\title{
Direction Preserving Zero Point Computing and Applications (Extended Abstract)
}

\author{
Xiaotie Deng ${ }^{1}$, Qi $\mathrm{Qi}^{2}$, Jie Zhang ${ }^{1}$ \\ 1 Department of Computer Science, \\ City University of Hong Kong, Hong Kong SAR, P. R. China \\ deng@cs.cityu.edu.hk, zhangjie3@student.cityu.edu.hk \\ 2 Department of Management Science and Engineering, \\ Stanford University, Stanford, California, USA \\ kaylaqi@stanford.edu
}

\begin{abstract}
We study the connection between the direction preserving zero point and the discrete Brouwer fixed point in terms of their computational complexity. As a result, we derive a PPAD-completeness proof for computing direction preserving zero point, and a matching oracle complexity bound for discrete Brouwer's fixed point.

Building upon the connection between the two types of combinatorial structures for continuous fixed point theorems, we derive an immediate proof that TUCKER is PPAD-complete for all constant dimensions, extending the results of Pálvölgyi for 2D case [20] and Papadimitriou for $3 \mathrm{D}$ case [21]. In addition, we obtain a matching algorithmic bound for TUCKER in the oracle model.
\end{abstract}

\section{Introduction}

Fixed point theorems have been fundamental in the literatures of Economics, such as equilibrium analysis of markets [1]. Fixed point computation has also been important in computational complexity study of equilibria. There is however a choice to make when we discretize the original concept of fixed point from the continuous domain.

Clearly, Sperner's simplex is a natural candidate for a discrete version of fixed point concept. Scarf, on the other hand, proposed a primitive set structure in his study of fixed point computation [23]. Iimura [13] defined the direction preserving function as a discrete version for the continuous function, and developed theorems for the existence of a zero point for any bounded direction preserving function.

The direction preserving function is defined on hypergirds, consisting of numerous hypercubes aligned along hyperplanes perpendicular to the coordinates. Informally, values of direction preserving functions will not have opposite signs on any pair of neighboring nodes. Based on such a concept in modeling continuous functions, Chen and Deng proved a matching algorithmic bound for approximate fixed point computation under the oracle complexity model [2]. 
Daskalakis, et al., on the other hand, developed a different discrete version of fixed point, defining it to be a hypercube on which all $d+1$ colors appear, for a hypergrid colored with $d+1$ colors. It allows the development that settled the computational complexity of Nash equilibrium computation $[8,5]$.

In this paper, we establish the equivalence of the two concepts in terms of their computation, both under the oracle function model, and the polynomial time computable function model. Therefore, it derives two immediate new results: PPAD-completeness for finding a zero point for direction preserving functions, and a matching algorithmic bound for finding a hypercube that contains all colors.

The computational equivalence of those two important models for discrete fixed point solutions has important implications. Based on the PPAD-complete result for zero point computation for direction preserving functions, we establish a PPAD-completeness proof of Tucker's problem for all constant dimensions. In the seminal paper of Papadimitriou introducing the PPAD class [21], he proved the PPAD-completeness of Tucker for 3D. It was also noticed that there is a similarity in difficulties to attempt PPAD-complete proofs for 2D SPERNER and 2D TUCKER. For 2D SPERNER, the difficulty was solved later by Chen and Deng [4]. The recent proof of PPAD-completeness for 2D Tucker by Pálvölgyi [20] exploits the techniques developed by Chen and Deng for 2D Sperner's PPAD proof. Our proof unifies the proof for all constant dimensions, through a modular approach. The connection also allows us to derive a matching oracle complexity bound for the computation of Tucker, for all constant dimensions.

The connection of DPZP and BROUWER requires a combinatorial structure that relates an integer function of values from 0 through $d$ to a direction preserving function of values $\left\{0, \pm e_{i}: i=1,2, \cdots, d\right\}$. For the oracle complexity of TUCKER, we develop a combinatorial parity lemma that relates the existence of complementary edges on the boundary with certain parity property of them in side the polyhedron.

Integer fixed point computation has important applications in Economics [13]. The PPAD-complete proof for direction preserving functions further reinforces our understanding of the correct complexity class it belongs to. The connection of the two different discrete fixed points may help in the studies of other related applications.

Tucker's lemma characterizes the fundamental combinatorial property underlying important mathematical problems such as the Borsuk-Ulam theorem, and Lovász's theorem on Kneser Graph (by Matoušek [18]). It has also applications to a type of the fair division problem called consensus-halving problem (Simmons and $\mathrm{Su}$ [24]) It has also been applied to the necklace problem and consensus- $\frac{1}{k}$-division problem (Longueville and Živaljević [16]).

In section 2, we review related concepts such as BROUWER, Direction Preserving Zero Point (DPZP for short) and TUCKER. In section 3, we prove DPZP is PPAD-complete and derive a matching bound for BROUWER. In section 4, we prove TUCKER is PPAD-complete for all constant dimension $d$, which extends the result for 2-D Tucker by [20] and 3-D Tucker by [21]. In section 5 , 
we derive an oracle computational matching bound for finding a complementary edge in TUCKER.

\section{Preliminaries}

In this section, we formally introduce three problems: the BROUWER, DPZP and the TUCKER.

Those three problems have solutions as guaranteed by mathematical theorems, often with an embedded parity argument. Complexities for finding such solutions of search problems are characterized by the classes of FNP and TFNP [19]. PPAD is a subclass of TFNP that is defined by a complete problem, the End of Line problem (or called LEFFE as in [3]). Several important fundamental problems are shown to be complete in this class, including SPERNER [21,4], NASH $[8,9,3,5]$, BROUWER, KAKUTANI, BORSUK-ULAM [21], approximate-NASH [6], Exchange Economy [21].

\subsection{BROUWER}

We should start with one class of those PPAD-complete classes, BROUWER, defined as follows.

Consider a $d$ dimension hypercube ( $d$-hypercube for short) of size $N$. A $d$ dimension hypergrid ( $d$-hypergrid for short) of scale $N$ places $N-1$ equally spaced hyperplanes parallel to each of the boundary faces of the hypercube and divides the hypercube into $N^{d}$ base hypercubes.

Let

$$
V_{N}^{d}=\left\{\mathbf{p}=\left(p_{1}, p_{2}, \cdots, p_{d}\right) \in \mathcal{Z}^{d} \mid \forall i: 0 \leq p_{i} \leq N\right\}
$$

Its boundary $B_{N}^{d}$ consists of point $\mathbf{p} \in V_{N}^{d}$ with $p_{i} \in\{0, N\}$ for some $i$ :

$$
B_{N}^{d}=\left\{\mathbf{p} \in V_{N}^{d}: p_{i} \in\{0, N\} \text { for some } i\right\} .
$$

For each $\mathbf{p} \in \mathcal{Z}^{d}$, let

$$
\mathcal{K}_{\mathbf{p}}=\left\{\mathbf{q}: q_{i} \in\left\{p_{i}, p_{i}+1\right\}\right\} .
$$

Let $m(\mathbf{p})=0$ if $\forall i, p_{i}>0$ and $m(\mathbf{p})=\min \left\{i: p_{i}=0\right\}$, otherwise. A color function $g: V_{N}^{d} \Rightarrow\{0,1, \cdots, d\}$ is valid if $g(\mathbf{p})=m(\mathbf{p}), \forall \mathbf{p} \in B_{N}^{d}$.

Definition 1. The input of BROUWER is a pair $\left(G, V_{N}^{d}\right)$ where $G$ generates a valid d-coloring $\mathbf{g}$ on $V_{N}^{d}$. That is, given any $\mathbf{p} \in V_{N}^{d}, G$ takes time polynomial in $N$ to compute the color $g(\mathbf{p})$ of a point $\mathbf{p}$.

The output of BROUWER is a point $\mathbf{p} \in V_{N}^{d}$ such that $\mathcal{K}_{\mathbf{p}}$ is fully colored, that is, $\mathcal{K}_{\mathbf{p}}$ has all $d+1$ colors.

It is known that BROUWER is PPAD-complete $[21,4]$ when $G$ is a polynomial time algorithm. As it is closely related to the Sperner's problem SPERNER [4], it is known to have a matching bound for the oracle model [2] as a result of the same lower bound for SPERNER [10]. 


\subsection{Direction Preserving Zero Point}

We should introduced another problem closely related to the fixed-point problem but for a restricted class of functions, the direction preserving functions.

Definition 2. A function $f: V_{N}^{d} \Rightarrow\left\{0, \pm \mathbf{e}_{i}, i=1,2, \cdots, d\right\}$ is direction preserving if for any $\mathbf{p} \in V_{N}^{d}, s, t \in \mathcal{K}_{\mathbf{p}}, f(s)+f(t) \neq 0$.

We call a direction preserving function $f$ on $V_{N}^{d}$ is bounded if $\forall \mathbf{p} \in V_{N}^{d}$, $\mathbf{p}+f(\mathbf{p}) \in V_{N}^{d}$.

We define the DPZP (Direction Preserving Zero Point) problem as follows:

Definition 3. The input of DPZP is a pair $\left(F, V_{N}^{d}\right)$ where $F$ computes the value $f(\mathbf{p})$ of a bounded direction preserving function for each $\mathbf{p} \in V_{N}^{d}$. That is, given any $\mathbf{p} \in V_{N}^{d}, F$ takes time polynomial in $N$ to compute the function value $f(\mathbf{p})$ of a point $\mathbf{p}$. The output of DPZP is a point $\mathbf{p} \in V_{N}^{d}$ such that $f(\mathbf{p})=0$, which is called a zero point.

It was known that a bounded direction preserving function $f$ on $V_{N}^{d}$ always has a zero point $[13,14]$, and there is a matching algorithmic bound [2] for the oracle model.

\section{$2.3 \quad$ TUCKER}

The complexity of TUCKER was first considered by Papadimitriou in [21], it was proved that TUCKER is in PPAD, and 3D TUCKER is PPAD-complete. The problem can be defined as follows:

Definition 4. (TUCKER) Consider the d dimension hypercube which is triangulated to be base simplexes. All vertices $V$ of the triangulated hypercube are colored by a function $f: V \Rightarrow\{ \pm 1, \cdots, \pm d\}$ and the boundary vertices are colored antipodal preserving, that is, $f(-\mathbf{v})=-f(\mathbf{v})$ for any boundary vertex $\mathbf{v}$. The problem is to find out a complementary 1-simplex in the hypercube, i.e., find out an edge in the triangulated hypercube so that the coloring of its two endpoints $\mathbf{p}, \mathbf{q}$ satisfying $f(\mathbf{p})=-f(\mathbf{q})$.

The existence of the solution for this problem is guaranteed by a combinatorial topology theorem, Tucker's lemma [26]. The original problem is built on a $d$ dimension ball, here we cast it into a $d$-hypercube to simplify of discussion.

\section{PPAD-completeness of Direction Preserving Zero Point}

In this section, we assume there is a polynomial time algorithm to compute $F$ in the DPZP problem. We prove DPZP is PPAD-complete and give a matching bound for BROUWER.

We will first introduce a reduction from DPZP to BROUWER. We then introduce a reduction from BROUWER to DPZP. As both reduction are polynomial time with respect to the algorithm $F$ and $G$, it shows that DPZP is 
PPAD-complete for the case $F$ is a polynomial time algorithm. Since DPZP has a matching algorithmic bound of $\theta\left(N^{d-1}\right)$ [2], it shows that BROUWER has a same matching algorithmic bound of $\theta\left(N^{d-1}\right)$.

Lemma 1. DPZP is in PPAD class.

Proof. We prove it in two steps. First we add one more layer to any given input of DPZP to achieve a specific boundary condition. Then we reduce the expanded DPZP to BROUWER.

Given an input of DPZP $\left(F, V_{N}^{d}\right)$, first we add one more layer on each face of its boundary which expands $V_{N}^{d}$ to $V_{N+2}^{d}$. Next we define a function $H$ : for each vertex $\mathbf{p} \in V_{N}^{d}, h(\mathbf{p})=F(\mathbf{p})$ and for any boundary point $\mathbf{p} \in V_{N+2}^{d} \backslash V_{N}^{d}$, either $h(\mathbf{p})=\mathbf{e}_{i}$ if $p_{i}=0$ and $\forall j<i: p_{j}>0$, or $h(\mathbf{p})=-\mathbf{e}_{i}$ if $\mathbf{p}>>0$ (all coordinates of $\mathbf{p}$ are positive), $p_{i}=N+2$ and $\forall j<i: p_{j}<N+2$. It is easy to verify that $\left(H, V_{N+2}^{d}\right)$ is still a DPZP.

Next, we reduce DPZP $\left(H, V_{N+2}^{d}\right)$ to $\operatorname{BROUWER}\left(G, V_{N+2}^{d}\right)$ as follows: define $g(\mathbf{p})=i$ if $H(\mathbf{p})=\mathbf{e}_{i} ; g(\mathbf{p})=0$ otherwise.

We illustrate the processes in Fig.1.

Clearly, a direction preserving with a bounded specific boundary condition function $h$ for DPZP translates into a valid coloring for BROUWER. For a solution of BROUWER, $\mathcal{K}_{\mathbf{p}}$, we should have $\mathbf{q} \in \mathcal{K}_{\mathbf{p}}$ such that $g(\mathbf{q})=0$. Then $h(\mathbf{q}) \in\left\{0,-\mathbf{e}_{1},-\mathbf{e}_{2}, \cdots,-\mathbf{e}_{d}\right\}$ by the process of our reduction. For $\mathbf{t} \in \mathcal{K}_{\mathbf{p}}$ and $g(\mathbf{t})=i \neq 0$, we have $h(\mathbf{t})=\mathbf{e}_{i}$. Since all colors appear in $\mathcal{K}_{\mathbf{p}}$, by direction preserving property of $h$, we must have $h(\mathbf{q})=0$, and hence $\mathbf{q}$ is a solution to DPZP.

\section{Lemma 2. DPZP is PPAD-hard.}

Proof. Given an input of BROUWER $\left(G, V_{N}^{d}\right)$, we define an input for DPZP on an expanded hypergrid. The expanded hypergrid obtained by placing $2 N$ more hyperplanes parallel to each of the boundary faces which refine each base hypercube in $V_{N}^{d}$ into $3^{d}$ smaller hypercubes.

After the reduction, one can easily check that:

(I) A valid coloring $g$ in BROUWER is translated into a bounded direction preserving function $f$ for DPZP;

(II) Once finding a zero point in DPZP, we can get a corresponding fixed point set in BROUWER.

Combining the above two lemmas, we have

Theorem 1. Direction Preserving Zero Point (DPZP) is PPAD-complete.

Since DPZP has a matching algorithmic bound of $\theta\left(N^{d-1}\right)$ [2], by the above reduction processes from DPZP to BROUWER and from BROUWER to DPZP, we obtain the following corollary.

Corollary 1. BROUWER has a matching algorithmic bound of $\theta\left(N^{d-1}\right)$. 


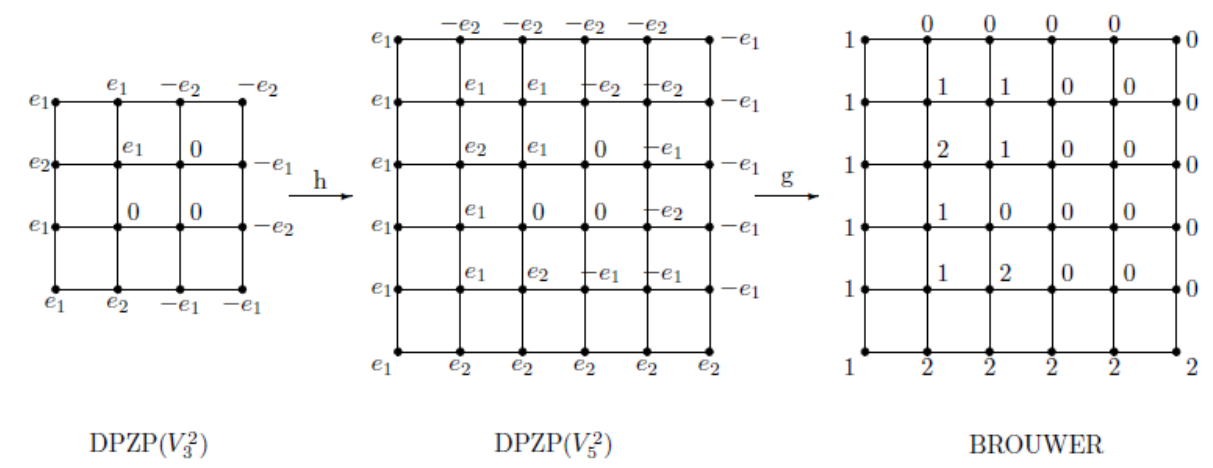

Fig. 1. The Proof of Lemma 1

\section{The Complexity of TUCKER}

In this section, we prove that $d$-D TUCKER is PPAD-complete for all constant dimension $d$, and hence extending the results for 2-D Tucker by [20] and 3-D Tucker by [21].

In [21], Papadimitriou used the path-following algorithm appeared in [12] to show TUCKER is in PPA, and stated that the orientation on complexes developed by Freund [11] can be used to prove TUCKER is in PPAD.

Lemma 3. [21] d-D TUCKER is in PPAD.

Next, we prove TUCKER is PPAD-hard for any dimension $d$. The reduction is based on DPZP and Kuhn's triangulation [15]. We should introduce Kuhn's triangulation first before giving the formal proof of PPAD-hardness.

Definition 5. (Kuhn's Triangulation) Let a d-hypercube of side length $\mathrm{N}$ be located in the first quadrant and one of its corner point at the origin, denote this vertex as $\mathbf{v}_{0}=(0,0, \cdots, 0)_{1 \times d}$ and its diagonal vertex as $(N, N, \cdots, N)_{1 \times d}$. We apply hyperplanes of side length 1 which parallel to coordinate axes to cut the big hypercube into $N^{d}$ unit d-hypercubes. Let $\mathbf{e}_{i}$ be a d-dimension vector that its $i$-th coordinate be 1 and others be 0 . Let $\pi=(\pi(1), \pi(2), \cdots, \pi(d))$ be any permutation of integers $0,1, \cdots, d-1$. Next we triangulate each unit hypercube in the following way: Let the vertex which is closest to $\mathbf{v}_{0}$ in each unit hypercube be the base point. Each permutation $\pi$ corresponds to one base simplex whose vertices are given by $\mathbf{v}_{\pi}^{i}=\mathbf{v}_{\pi}^{i-1}+\mathbf{e}_{\pi(i)}$, where $\mathbf{v}_{\pi}^{0}$ is the base point of that unit hypercube. Then each of the unit hypercube is triangulated to d! base simplexes.

We illustrate Kuhn's triangulation for a 3-dimension hypercube with side length 1 in Fig. 2. It is easy to see that all vertices of the base simplexes are the vertices of the hypercubes. We claim that these simplexes all have disjoint 


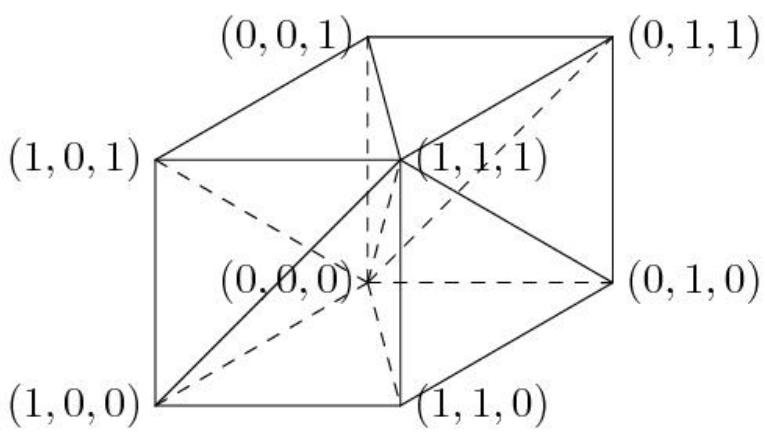

Fig. 2. Kuhn's triangulation in 3 dimension

interiors and the union of them is the $d$-hypercube. In fact, Kuhn's triangulation can be acquired by an equivalent cutting approach, that is, first using the $d$ ! permutations $\pi$ to triangulate the big hypercube to $d$ ! simplexes, and then triangulate each big simplex into $N^{d}$ base simplexes.

Lemma 4. $d$-D TUCKER is PPAD-hard.

Proof. To prove TUCKER is PPAD-hard, we reduce DPZP to it. For any input of DPZP $\left(F, V_{N}^{d}\right)$, we first add one more layer to make DPZP $\left(H, V_{N+2}^{d}\right)$ satisfy the antipodal constraint for the function values. This can be done by defining $h$ as: for each vertex $\mathbf{p} \in V_{N}^{d}, h(\mathbf{p})=F(\mathbf{p})$ and for any boundary point $\mathbf{p} \in$ $V_{N+2}^{d} \backslash V_{N}^{d}$, either $h(\mathbf{p})=\mathbf{e}_{i}$ if $p_{i}=0$ and $\forall j<i: N+2>p_{j}>0$, or $h(\mathbf{p})=-\mathbf{e}_{i}$ if $p_{i}=N+2$ and $\forall j<i: 0<p_{j}<N+2$. It is easy to verify that $\left(H, V_{N+2}^{d}\right)$ is still a DPZP.

Next we reduce DPZP $\left(H, V_{N+2}^{d}\right)$ to TUCKER $\left(G, V_{N+2}^{d}\right)$ by defining function $g$ as: $g(\mathbf{p})=i$ if $h(\mathbf{p})=\mathbf{e}_{i} ; g(\mathbf{p})=-i$ if $h(\mathbf{p})=-\mathbf{e}_{i} ; g(\mathbf{p})=-1$ if $h(\mathbf{p})=$ 0 . Obviously, the function values of $g$ satisfy TUCKER's antipodal boundary condition.

The last step is to triangulate each small hypercube by Kuhn's triangulation. Since Kuhn's triangulation will not add any extra vertices, the output of Kuhn's triangulation combining with the function $g$ is an instance of TUCKER $\left(G, V_{N+2}^{d}\right)$. By TUCKER's lemma, it has a complementary edge. Because of the direction preserving property of the original function $h$, the complementary edge has to be $(1,-1)$. Again because of the direction preserving property, that node $\mathbf{p}$ with value -1 in the complementary $(1,-1)$ must corresponding to $h(\mathbf{p})=0$ in the original problem. The result follows. We illustrate the reduction process in Fig. 3.

Theorem 2. $d-D$ TUCKER is PPAD-complete. 


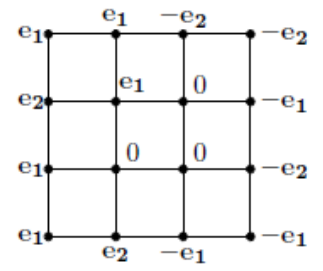

$\operatorname{DPZP}\left(V_{3}^{2}\right)$

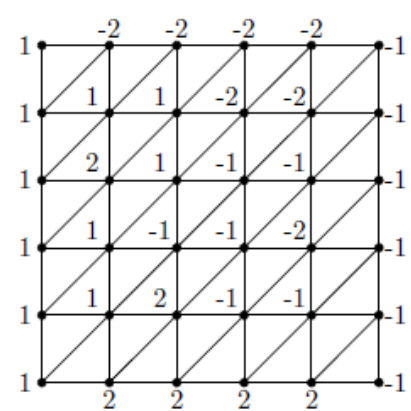

TUCKER

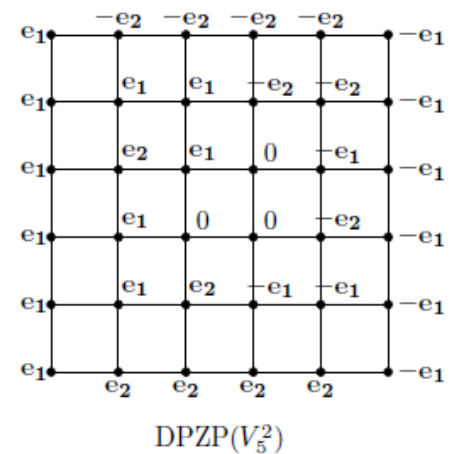

!

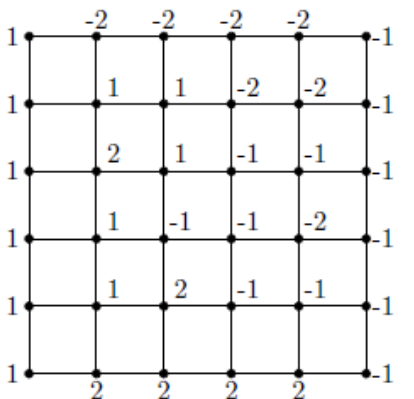

Fig. 3. The Proof of Lemma 4

\section{Matching Bound of TUCKER in Oracle Model}

In this section, we derive the orale matching bound for TUCKER.

Theorem 3. (Lower Bound) For any instance of d-D TUCKER, one can find the complementary 1-simplex in time $\Omega\left(N^{d-1}\right)$.

Proof. Using the same reduction as in PPAD-hardness proof of Tucker, we notice that if there is an algorithm that solves a problem of size $N^{d}$ for TUCKER, it solves a problem of size $N^{d}$ for DPZP. Since there is a lower bound of $\Omega\left(N^{d-1}\right)$ of DPZP [2], it results in a same lower bound $\Omega\left(N^{d-1}\right)$ for TUCKER.

The upper bound can be derived in two steps. First, we check if there is a complementary edge on the boundary. This takes time complexity $O\left(N^{d-1}\right)$. If the answer is no, we prove that the number of $(d-1)$-simplexes which are fully colored on the boundary must be odd. Then by using binary search based on the parity argument, one can find a complementary edge in time $O\left(N^{d-1}\right)$. Hence, the total time complexity is $O\left(N^{d-1}\right)$.

For $d=2$, the following boundary lemma is easy to check. 
Lemma 5. For any instance of $2 D$ TUCKER, if there is no complementary edge on the boundary, then the number of $\{1,2\}$ edges on the boundary must be odd.

For higher dimensions, we employ the parity argument in Cohen's proof of Tucker's lemma [7] to prove the boundary lemma. Due to the huge enumeration of different dimension simplexes, we prove the 3D case for illustration.

Lemma 6. For any instance of 3-D TUCKER, if there is no complementary edge on the boundary, then there must exist one kind of $\{a, b, c\}$-simplex that its number is odd, where $a, b, c$ are of different signs and their absolute value are distinct.

Proof. For contradiction, we assume that the number of all $\{a, b, c\}$-simplexes on the boundary are even. Let $A$ and $A^{\prime}$ be the center of two opposite faces. Let $P$ be a path on the boundary from $A$ to $A^{\prime}$, and $P^{\prime}$ is the antipodal path of $P$. Then $P$ and $P^{\prime}$ separate the boundary of the cube to two parts. Let us denote one part as $S$ and the other part denoted as $S^{\prime}$.

Let $S(a, b, c)$ be the number of $\{a, b, c\}$-simplex on $S$, and $S^{\prime}(a, b, c)$ be the number of $\{a, b, c\}$-simplex on $S^{\prime}$. According to the assumption, we have

$$
S(a, b, c)+S^{\prime}(a, b, c)=0 \bmod 2
$$

since the number of $\{a, b, c\}$-simplex on $S^{\prime}$ is equal to the number of $\{-a,-b,-c\}$ simplex on $S$, i.e.,

$$
S^{\prime}(a, b, c)=S(-a,-b,-c)
$$

we get

$$
S(a, b, c)+S(-a,-b,-c)=0 \bmod 2
$$

On the other hand, let $P(a, b)$ denotes the number of $\{a, b\}$ edges on $P$. As $A$ and $A^{\prime}$ are of different signs, $P$ must has odd number of $\{a, b\}$ edges where $a$ and $b$ possess different signs. Since there is no complementary edge on the boundary, there are six possible this kind of edges, i.e., $\{1,-2\},\{1,-3\},\{2,-1\},\{2,-3\},\{3,-1\}$ and $\{3,-2\}$. So, we have

$$
P(1,-2)+P(1,-3)+P(2,-1)+P(2,-3)+P(3,-1)+P(3,-2)=1 \bmod 2
$$

Now we going to derive a contradiction by parity argument. See Fig.4 for illustration.

Take $S$ into consideration, and consider the 2 -simplexes on the boundary starting from edges $\{1,-2\}$ lying on $P \bigcup P^{\prime}$, since there is no complementary edge on $S$, those $\{1,-2\}$ edges will either reach another $\{1,-2\}$ edge on $P \bigcup P^{\prime}$, or will terminate with a $\{1,-2, \pm 3\}$-simplex on $S$. Starting from $\{1,-2\}$ edges lying on $S \backslash\left(P \bigcup P^{\prime}\right)$, they will be pairwised in two $\{1,-2, \pm 3\}$ simplexes, or one in a $\{1,-2, \pm 3\}$ simplex while another on $P \bigcup P^{\prime}$. This gives a parity argument that

$$
S(1,-2,3)+S(1,-2,-3)=P(1,-2)+P^{\prime}(1,-2) \bmod 2
$$




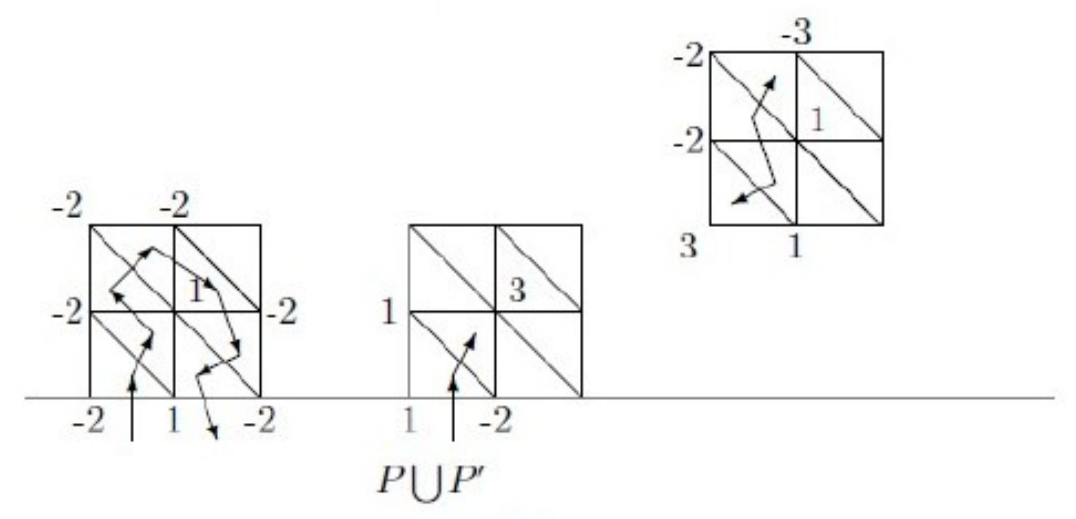

Fig. 4. The proof of Lemma 6

which implies

$$
S(1,-2,3)+S(1,-2,-3)=P(1,-2)+P(-1,2) \bmod 2
$$

Similarly, we have

$$
S(1,2,-3)+S(-1,2,-3)=P(2,-3)+P(-2,3) \bmod 2
$$

and

$$
S(-1,2,3)+S(-1,-2,3)=P(-1,3)+P(1,-3) \bmod 2
$$

Summing up these three equations we get a contradiction with the assumption, that gives a proof to the lemma.

We can transfer the kind of $\{a, b, c\}$-simplex whose number is odd to $\{1,2,3\}$ simplex as follows: for example, if it is $\{1,-2,3\}$-simplex whose number is odd, then we exchange labels of 2 and -2 , then the numbers of $\{1,-2,3\}$-simplexes and $\{1,2,3\}$-simplexes are exchanged and this process does not affect the complementary edges in the instance.

Proposition 1. If there is no complementary edge on the boundary, then the number of $\{1,2, \cdots, d\}$-simplexes on the boundary of d-dimensional hypergrid triangulated by Kuhn's triangulation has the same parity as the number of $\{-i, 1,2, \cdots, d\}$ simplexes, $i=1,2, \cdots, d$, in the hypergrid.

Proof. We change the labels of all nodes with negative labels to zero. Then the claim follows from standard theorems on indexes(see, e.g., [2]).

Theorem 4. (Upper Bound) The upper bound of d-TUCKER is $O\left(N^{d-1}\right)$ under Kuhn's triangulation when the utility functions are given by oracle. 
Proof. First, by checking boundary we can see whether there is a kind of $\left\{v_{1}, v_{2}, \cdots, v_{d}\right\}$ simplex whose number is odd on the boundary. If not, there must exist complementary edges on the boundary, finding it can be completed in time $O\left(N^{d-1}\right)$. If we did not find any complementary edge on the boundary, we relabel all nodes of negative labels by changing them to 0 . Then the divide- $\&$-conquer approach applies. We will end with a simplex of labels $\{0,1, \cdots, d\}$. As 0 was transferred from some negative labelled node, say one labelled with $-i$. Then $(i,-i)$ in this simplex will be the complementary edge we set off to find.

Since the size of the hypercube decrease geometrically, the complexity of checking which case occurred will not exceed $O\left(N^{d-1}\right)$.

Theorem 5. For any d-hypercube which is an instance of TUCKER and it is triangulated by Kuhn's triangulation, the complementary 1-simplex can be found in time $\theta\left(N^{d-1}\right)$ under oracle model.

\section{Conclusion}

Our study builds a computational complexity connection between two types of discrete versions of fixed point concepts, which implies two new results: a PPADcompleteness proof for computing direction preserving zero point, and a matching oracle complexity bound for discrete BROUWER's fixed point. Furthermore, the connection allows for a clear proof that TUCKER is PPAD-complete for all constant dimensions, that extend the results of Pálvölgyi for 2D case [20] and Papadimitriou for 3D case [21]. At the same time, a matching algorithmic bound for TUCKER is obtained in the oracle model.

As fixed point analysis is fundamental for many economic equilibrium problems, our study, though purely out curious minds for the fundamentals, could be of application values for other algorithmic game theory problems, in one way or another. We strongly believe that simplicity carries a value in its own right in theory building, and could play an important role in the development of a field. We demonstrate this value by presenting a simple and clean proof of complexities of TUCKER, both in the oracle function model and the polynomial time function model.

\section{References}

1. K. Arrow and G. Debreu, Existence of an equilibrium for a competetive economy. Econometrica, 22:265-290, 1954.

2. X. Chen and X. Deng. On algorithms for discrete and approximate brouwer fixed points. STOC 2005. 323-330. Journal version appeared at: X. Chen and X. Deng: Matching algorithmic bounds for finding a Brouwer fixed point. J. ACM 55(3): (2008)

3. X. Chen and X. Deng, 3-NASH is PPAD-Complete,Electronic Colloquium on Computational Complexity (ECCC)(134):(2005)

4. Xi Chen, Xiaotie Deng: On the Complexity of 2D Discrete Fixed Point Problem. ICALP (1) 2006: 489-500. 
5. X. Chen and X. Deng, Settling the Complexity of Two-Player Nash Equilibrium. FOCS 2006: 261-272

6. X. Chen, X. Deng and Shang-Hua Teng, Computing Nash Equilibria: Approximation and Smoothed Complexity. FOCS 2006: 603-612

7. D. I. A. Cohen. On the Combinatorial Antipodal-Point Lemmas. Journal of Combinatorial Theory, Series B, 87-91, (27) 1979.

8. C. Daskalakis, P. W. Goldberg and C. H. Papadimitriou, The complexity of computing a Nash equilibrium, STOC, 2006

9. C. Daskalakis and C. H. Papadimitriou, Three-Player Games Are Hard. Electronic Colloquium on Computational Complexity (ECCC)(139): (2005)

10. X. Deng, Q. Qi and A. Saberi, On the Complexity of Envy-Free Cake Cutting, http://arxiv.org/abs/0907.1334.

11. R. M. Freund, Variable dimension complexes. Part I: Basic theory, Math. Oper. Res.9, No.4, 479-497, 1984.

12. R. M. Freund and M. J. Todd, A constructive proof of Tucker's combinatorial lemma, Journal of Combinatorial Theory, Series A, 321-325, 30(3) 1981.

13. T. Iimura. A discrete fixed point theorem and its applications. Journal of Mathematical Economics, 39:725-742, 2003.

14. T. Iimura, K. Murota, and A. Tamura. Discrete fixed point theorem reconsidered. METR, 92004.

15. K. W. Kuhn, Some combinatorial lemmas in topology. IBM J. Res. Dev 4(5), 518-524, 1960.

16. M. Longueville and R.T. Živaljević. The Borsuk-Ulam-property, Tucker-property and constructive proofs in combinatorics. Journal of Combinatorial Theory, Series A, 839-850, (113)2006.

17. J. Matoušek. Using the Borsuk-Ulam Theorem, Springer-Verlag Berlin Heidelberg New York, 2003.

18. J. Matoušek, A combinatorial proof of Kneser's conjecture, Combinatorica 24(2004) $163-170$

19. N. Megiddo and C. H. Papadimitriou: On Total Functions, Existence Theorems and Computational Complexity. Theor. Comput. Sci. 81(2): 317-324 (1991).

20. D. Pálvölgyi, 2D-TUCKER is PPAD-complete, Preprint in ECCC

21. C.H. Papadimitriou. On the complexity of the parity argument and other inefficient proofs of existence. Journal of Computer and System Sciences, 498-532, 1994.

22. T. Prescott and F. E. Su, A constructive proof of Ky Fan's generalization of Tucker's lemma, Journal of Combinatorial Theory, Series A, 257-265, (111)2005.

23. H. E. Scarf, The approximation of fixed points of a continuous mapping. SIAM J. Applied Mathematics 15, 997-1007, 1967.

24. F. W. Simmons and F. E. Su, Consensus-halving via theorems of Borsuk-Ulam and Tucker, Mathematical Social Science, 15-25, (45)2003.

25. F. E. Su, Rental Harmony: Sperner's Lemma in Fair Division,Amer. Math. Monthly, 930-942, (106)1999.

26. A. W. Tucker, Some topological properties of disk and sphere, Proceedings of the First Canadian Mathematical Congress, 285-309, 1945.

27. G. M. Ziegler, Lectures on Polytopes, Graduate Texts in Mathematics, vol.152, Springer, New York, 1995. 